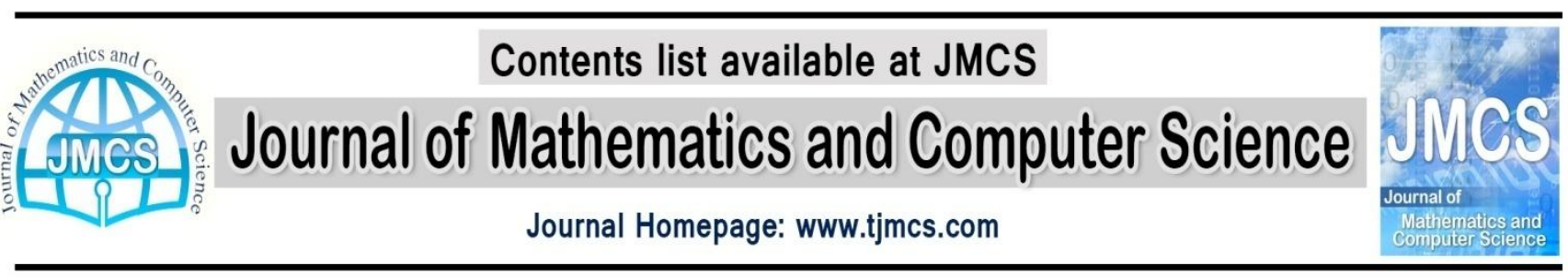

\title{
Attendance Checking System Using Quick Response Code for Students at the University of Sulaimaniyah
}

\author{
Miran Hikmat Mohammed Baban \\ Computer Dept. of the Science Faculty, Sulaimaniyah University, Sulaimaniyah KRG-Iraq
}

miran.baban@ymail.com

Article history:

Received March 2014

Accepted April 2014

Available online April 2014

\begin{abstract}
A system to record and view attendance information using a QR code scanned by smart phones is proposed to help students avoid penalties that may result from poor attendance. In proposed system, students can easily view their attendance records for each course. The proposed system was modeled and developed for use at the University of Sulaimaniyah; however, it could be applied to other schools and colleges.
\end{abstract}

Keywords: QR-Code Scanning, PHP, MYSQL, MAC Address, Database Connection, Absentee rate, Smart Phones, Microsoft Excel sheet.

\section{Introduction}

\subsection{Attendance checking system}

The most important factor for students in schools and universities is regular attendance. Students who are absent from lectures will experience issues obtaining additional instruction and information from their teachers. As a result of significant absenteeism, students may more likely to be unemployed, homeless, or involved in justice system. Students with high rates of absenteeism can affect other students who have regular attendance in the same class. High rates of absenteeism may cause students to fail in a specific classand they may have to enroll another school or university. Therefore, universities should provide a system that helps students to improve attendance [9].

Attendance systems are used in many universities. At the University of Sulaimaniyah an attendance system is widely used by both colleges and departments. Typically, the attendance system is managed by each teacher. Teachers record a list of students that are absent from class or lectures. 
Moreover, some teachers at the University of Sulaimaniyah make attendance a part of a student's grade; therefore to obtain the best grades possible, students must attend classes. However, some students are not aware of how many classes they have missed because this information can be difficult to obtain,

Therefore, students with high rate of absenteeism may receive low grades or may fail a class. For example, the College of Science has mandated that all students be attends all lectures.

\subsection{QR code}

A Quick Response code (QR- code) is a two dimensional bar code designed by Denso Wave in 1994 in Japan. A QR code is arranged in rows and columns of black and white, and has been designed to be read by smart phone [1].

QR code can hide large amount of data, numeric and alphanumeric. Thus, they have become popular all over the world. Moreover, QR codes are widely used in telecommunication due to increased popularity of smart phones, which typically contain software that can read QR-code images [2].

A QR-code image comprises a functional pattern and an encoding region as can be seen in Fig. 1. The patterns included in a QR-code image are finder, alignment, timing, and separator patterns. Each of these patterns has its own functionality [6].

1. Finder pattern: This pattern can be found at the edges of a $Q R$ code image. The finder pattern is a square block that contains that contains a black square. There are three finder patterns on every QR code image; at the top left, top right, and bottom left. There is not finder pattern at the bottom right.

The primary functionality of the finder pattern is to tell a scanner or decoder that the image that has been encoded as a QR-code image. No data is stored in the finder pattern.

2. Alignment pattern:Similar to the finder, there is no data stored in the alignment pattern; however, it provides information scanner devices to correctly position the data stored in the encoded data region. The alignment pattern is positioned between encoded data and is usually in the center of the image.

The structure of this pattern consists of a small square with a tiny dot inside. In addition, the number of alignment patterns can differ for different $\mathrm{QR}$ codes.

3. Timing pattern:This pattern lies between two finder patterns. Timing patterns are arranged both vertically and horizontally. There is a black dot inside each timing pattern.

The main purpose of the timing pattern is to correct the central coordinate for each data cell when any distortion occurs during decoding of symbols or when an error is found in any cell pitch in the QR code. No data is stored in the timing pattern.

4. Encoded data:This pattern is located at the center of the image. Data is stored within this pattern. In addition, when data is inserted, it is converted to binary data. This binary data is converted back to the normal text when the image is decoded by a scanner [7]. 


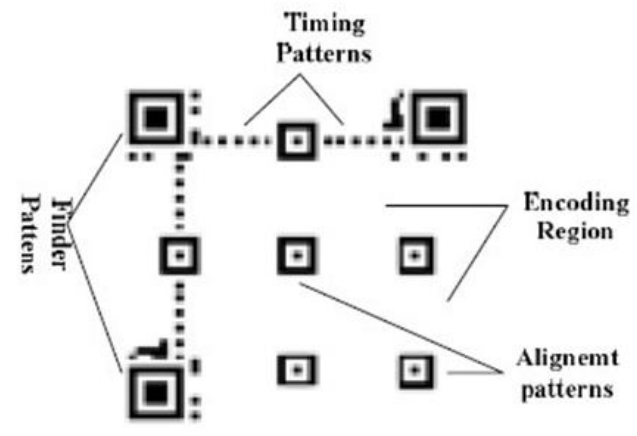

Figure 1. Structure of QR Code [6]

\subsection{Smart Phones}

Smart Phones have become one of the most intelligent consumer devices. They can perform many functions, such as organizing personal data, taking photos and voice telephony [3].

Recently, the functionality of smart phones has grown to cover some of the functionality of personal computers in both professional and social contexts [4]. In addition, smart phones are portable communication devices that work to connect and integrate telecommunication and internet services in a single device. Furthermore, smart phones can be used for network communication between different devices [5].

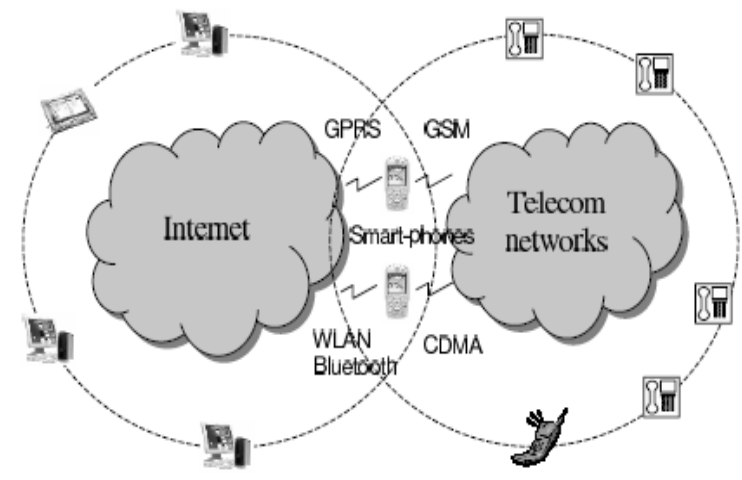

Figure 2. Smart phones can be end-points between the internet and telecommunication networks [5]

This paper will discuss how the current attendance system works at the University of Sulaimaniyah and will discuss the current system's disadvantages. In addition, this paper will demonstrate the advantages of the proposed system and will discuss the plans for creating the proposed system, including explanations of the techniques and mechanisms employed. 


\section{System requirements}

\subsection{Hardware requirements}

The system requires that the university provide a server to process university data and install the required software.

\subsection{Software requirements}

This system requires a database (MySQL) to store information about students, teachers, colleges, departments, and attendance. Moreover, the proposed system requires PHP and Apache to create web pages, which are used to load and upload data from the user to the server and vice versa.

In the proposed system, students scan QR codes using mobile phones to obtain their attendance information. The QR codes are generated by teachers and the attendance system administrator using software and web sites.

"QRreader" and "Bar capture" are software packages that can generate QR codes.

Websites that can generate QR Codes:

- http://www.qrstuff.com/

- http://www.qrcode-monkey.com/

- http://createqrcode.appspot.com/

- http://www.unitaglive.com/arcode

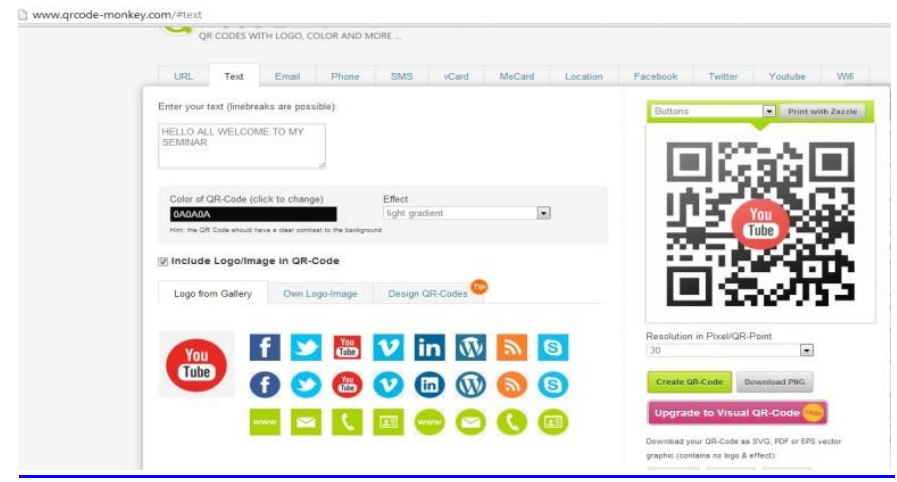

Figure 3 (a) QRCode-Monkey website for generating QR codes 


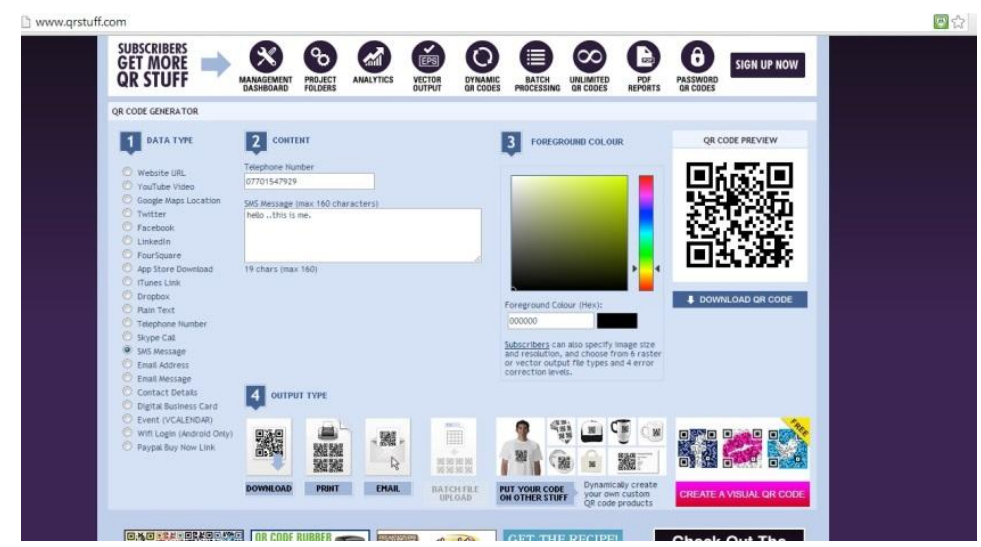

Figure 3 (b) QRCode-Stuff website for generating QR codes

\section{User requirements}

\subsection{Hardware requirements}

This system requires that each user has a camera-enabled smart phone that is capable of scanning QR codes. In addition, computers must be available for administrators and teachers to insert attendance information to the database.

\subsection{Software requirements}

The proposed system requires that each student's smart phone is capable of reading QR code images. QR code software is available on mobile application marketplaces, such as Apple's App Store and Google's Play Store.

\section{How the current system works}

The following outlines how the current system works.

1. A teacher of a specific subject reads the names of students from a list.

2. If there are any absentees, the teacher records each absent student by putting an " $\mathrm{A}$ " behind their names ("A" denotes "Absent").

3. After checking the attendance list, the teacher enters each absent student's name on a small paper form.

4. The attendance form is then passed to the system manager. The primary role of the system manager is to double check the list and enters all absentee information in a Microsoft Excel sheet. 
5. To calculate the absentee rate, the system manager performsmanual calculationsto count the number of absences for each student. Then, they re-enter the data.

6. The system manager then posts a warning list for students on a notice board.

\section{Current system problems}

The following outlines the problems with the current system.

1. The process is performed very slowly.

2. The paper form can be lost by teachers. This leads to mistakes in absentee rates.

3. In some cases, teachers have problems writing student names clearly (e.g., bad handwriting)(Fig. 4 (a)).This leads to difficulty for the system manager when entering data.

4. The form is sometimes incomplete, which means some fields cannot be completed;e.g.,date, subject name, teacher name, etc.

5. All data (paper forms) are entered to a Microsoft Excel file at the end of each class. This results in significant labor for the system manager.

6. The system manager may make mistakes when calculating the absentee rate for each student, which could result in an unwarranted failing grade for a specific module. However, the calculation process is quite involved because there is a significant amount of addition, division, and multiplication.

7. Students may not see the notice board that announces attendance warnings.

8. The paper form may not be sufficiently large to record all absent students' names (Fig. 4 (b)).

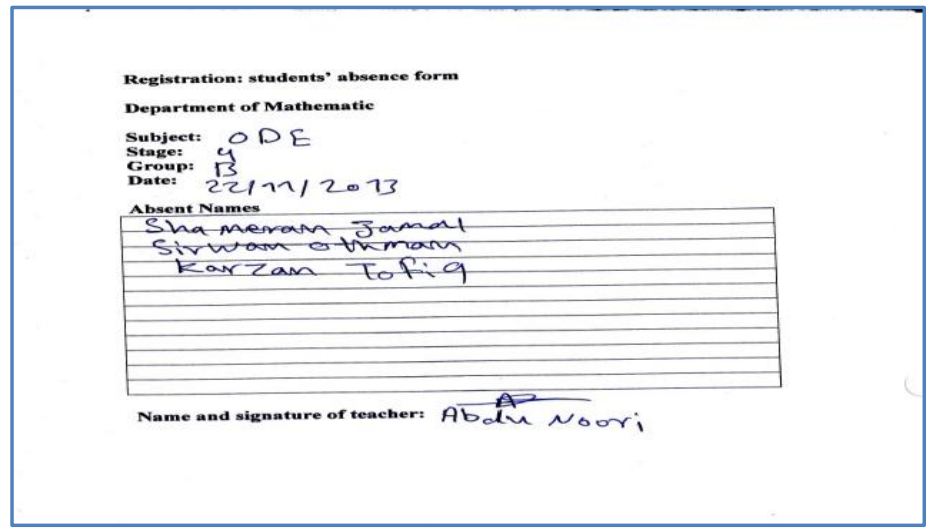

Figure 4 (a). Bad hand writing on absentee form 


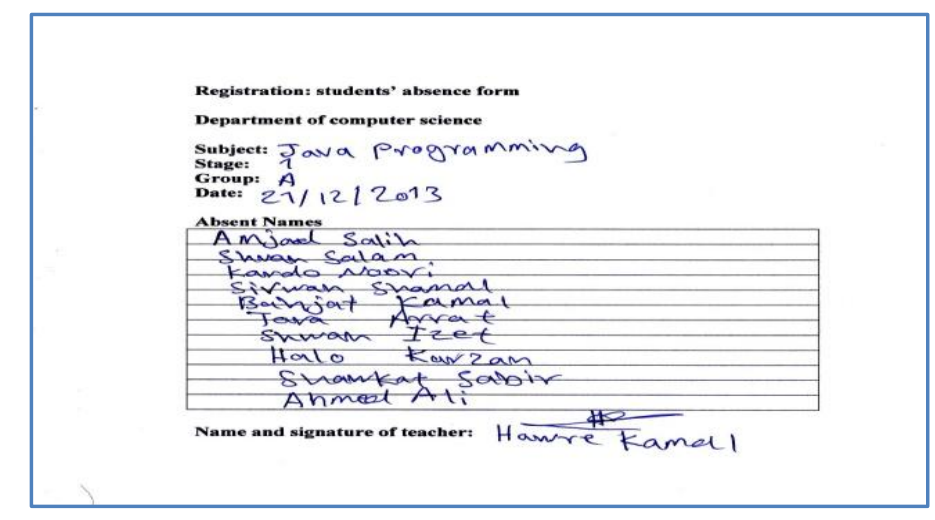

Figure 4 (b). Small absentee form

\section{How the new system will work}

The proposed system will replace the old system completely. All functions will be computerized and will involve the following general steps.

A Each teacher will receive an Excel file that contains student names(Fig. 5).

A Teachers read student names to check attendance.

With every absence, the teacher will mark a box for each absent student to identify that student as absent on a specific date.

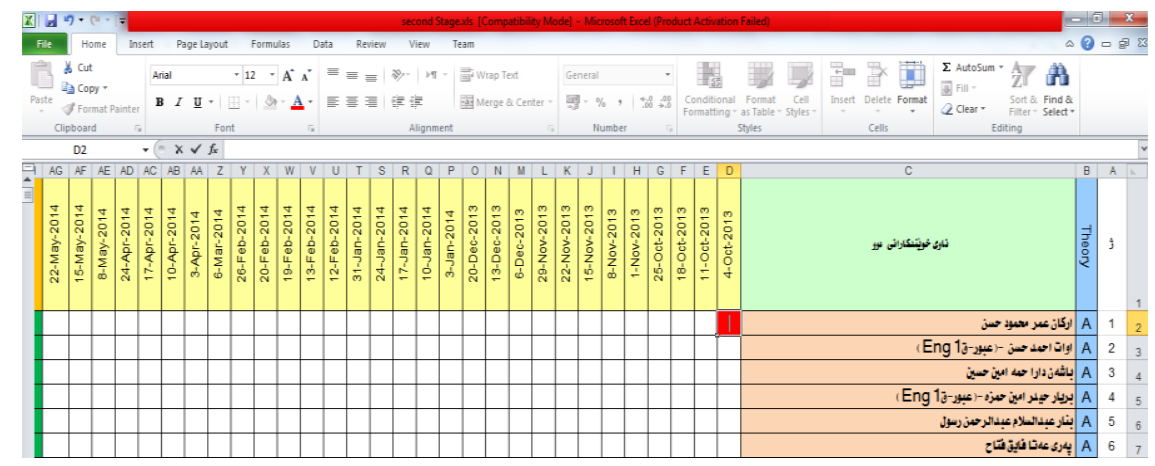

Figure 5. Excel sheet for checking class attendance

A Teachers send the Excel document to the administrator.

A The administrator enters the names of the absent students to the MySQL database.

A Each week, teachers place a QR code image on their doors that students can scan using a smart phone. This QR code contains a URL that links to a web page that displays the student's absentee rate. Figure 6 shows an example of a QR code. 


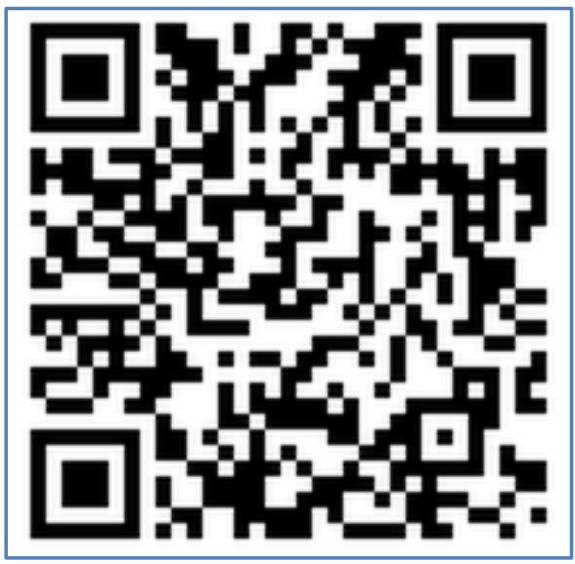

Figure6. Example QR code

A Using the QR codes, students can regularly determine their absentee rate for each lecture and course.

If any student has a complaint about the reported absentee rate, they can contact the system administrator or their teacher to investigate the matter.

\section{Advantages of the new system}

A The calculation of the absentee rate is easier and more accurate; all calculations are performed by computer rather than manually.

A There will be no paper work involved; all processes are performed by computer. This will help protect against loss of data.

A The computerized processes will be performed more quickly and accurately than the previous system.

A The proposed system eliminates the notice board. Absentee rates can be viewed weekly by scanning an easily accessible QR code.

All data is saved to a university database server and an Excel sheet. This will allow for easy data monitoring.

\section{Student authentication}

To reach any system that contains sensitive user data, there must be some sort of authentication method that allows users to access the system securely. The login process depends on the type of security methods 
the system provides. For example, systems can control access with passwords, biometric authentication, or authentication certificates [2].

In the old system, there is no security provided. In addition, the old system makes all absentee information public. However, some students may wish to keep their attendance records private. The proposed system provides a high level of security and privacy. The proposed system provides a high level of security and privacy. The system can protect personal student data as URL inside a QR code image, which prevents data from unwanted publication.

Only registered students can scan the QR code, and the results of the scan will show only the individual student's absentee data.

This security will be performed using the students' smart phone Media Access Control (MAC) address, which is preregistered to the proposed system. When a student scans the QR code image, they are authenticated using their device's MAC address.

\section{Conclusion}

This paper has presented a new system that will allow students at the University of Sulaimaniyahto determine their absentee rate regularly. The proposed system will organize and store all data in a database server; thus, the data will be secure and protected against loss.

Using the proposed system, students will be able to determine their monthly attendance information accurately using smart phones to scan a QR code. In addition, the proposed system will reduce both administrative manual labor and paper requirements because all of the system's processes are computerized.

\section{References}

1. D.-H. Shin, J. Jung, B.-H. Chang, The psychology behind QR codes: user experience perspective, Computers in Human Behavior 28 (2012), 1417-1426.

2. I.C. Zupanovic and E. Tijan, QR Codes as a time management tool in m-learning, Proc. MIPRO 2012, Opatija, Croatia, $1470-1474$

3. M.M. Swarup, A. Dwivedi, C. Sonkar, R. Prasad, M. Bag, V. Singh, A QR code based processing for dynamic and transparent seat allocation in Indian railway, Int. J. Comp. Sci. 9 (2012) 338-344.

4. Y.-G. Kim and M.-S. Jun, A design of user authentication system using QR code identifying method, Proc. ICCIT 2011, Seogwipo, South Korea, 31-35.

5. C. Guo, H.J. Wang, W. Zhu, W, Smart-phone attacks and defenses, HotNets III, November, (2004), San Diego, CA.

6. Y. Liu, J. Yang, M. Liu, Recognition of QR code with mobile phones, Proc. CCDC 2008, Yantai, Shandong, $203-206$.

7. A.L. Hou, F. Yuan, G. Ying, QR code image detection using run-length coding, Proc. ICCSNT 2011, Harbin, China, 2130-2134. 
M. H. M. Baban / J. Math. Computer Sci. 10 (2014), 189-198

8. I. Kapsalis, Security of QR Code, Msc. Norwegian University of Science and Technology, Department of Telematics (2013).

9. S. Rothman, School absence and student background factors: A multilevel analysis, International Education Journal 2 (2001) 59-68. 